(C) 1982. The Genetical Society of Great Britain

\title{
DYNAMICS OF THE B-CHROMOSOME POLYMORPHISM IN RYE. I. SIMULATED POPULATIONS
}

\author{
R. B. MATTHEWS and R. N. JONES \\ Department of Agricultural Botany, \\ University College of Wales, Aberystwyth, U.K.
}

Received 28.vii.81

\begin{abstract}
SUMMARY
A computer model has been developed to simulate the transmission characteristics of the $B$ chromosomes in rye, and to provide a basis on which to identify, and to understand, the main factors responsible for determining the equilibrium $B$-frequency levels in open pollinating populations. The model has been devised by expressing the behaviour of the $B$ s, at various phases of the life cycle, in terms of mathematical equations. These equations contain parameters which determine the behaviour of the $B$ s at meiosis, pollen grain (and egg cell) mitosis and during the development of gametes and sporophytes.

By exploring a range of values for the parameters it has been possible to ascertain that variation in the amount of meiotic elimination of $B \mathrm{~s}$, as well as in their direction rate during nondisjunction in the pollen grain and egg cell, influence the final $B$-frequency equilibrium. Variation in the rate of nondisjunction on the other hand, affects the number of generations required to attain equilibrium more than the final equilibrium itself. Selection against gametes and plants containing $B \mathrm{~s}$, even at levels which are higher than can reasonably be expected to occur naturally, cannot prevent the accumulation of $B$ s within populations, provided that high rates of directed nondisjunction are also occurring.

The results are discussed in relation to the $B$ chromosome polymorphism in natural populations of rye.
\end{abstract}

\section{INTRODUCTION}

IN wild populations and primitive cultivated strains of rye there is an extensive polymorphism for the presence of supernumerary $B$ chromosomes (Müntzing, 1950, 1957a, $b$; Kranz, 1963; Lee and Min, 1965a, $b$; Zecěvić and Paunović, 1967). Japanese populations have a $B$ frequency in excess of 90 per cent, with the majority of individuals having $2 B \mathrm{~s}$ (Kishikawa, 1965). The range of $B$ chromosomes usually extends from $0-4$, with higher numbers than this, and with odd-numbered combinations being present in very low frequencies.

The frequency, and the pattern of distribution, of the $B$ chromosomes varies widely between populations of different origin and habitat and the reasons for this variation are not understood. $B$ chromosomes could have arisen, or could have been introduced, into different populations at different times. No datum is available on the change in frequency over a number of generations and we have no idea which, if any, of the populations studied are at equilibrium. It has not been possible either to associate the incidence or distribution of $B \mathrm{~s}$ with any particular environmental variable, or to demonstrate any influence due to natural selection which may be significant in determining the pattern of the polymorphisms. 
It is known of course that, given the existence of $B$ chromosomes within a population, the polymorphism can be generated and maintained by the very nature of the mode of inheritance of the $B$ s themselves. They are mitotically stable in rye and the same number of $B s$ is found throughout the somatic tissues of the sporophyte. At meiosis they pair exclusively among themselves and are distributed effectively into the products of both male and female meiosis-notwithstanding the complications due to polysomy and some loss when they occur as univalents. Their unusual and nonmendelian behaviour is manifested at the first mitosis in the gametophytes. At first pollen grain mitosis they undergo directed nondisjunction and are included, in an unreduced number, in the generative nucleus-and thereby into the sperm nuclei. An equivalent act of self preservation takes place on the female side leading to their inclusion in unreduced numbers in the egg cell. This property of directed nondisjunction not only ensures their survival in the progeny but also boosts their frequency, in even numbered combinations, over successive generations of open pollination. The effectiveness of $B$ chromosome transmission through meiosis, and through the gametophyte phase as well, varies for different populations, and is, to a large extent, a property of the particular $B$ present in a given population (Jones, 1975). The structure of $B$ chromosomes, as judged from an analysis of the chromomere pattern at pachytene, also varies for $B \mathbf{s}$ derived from diverse sources (Lima-De-Faria, 1963).

A further complicating factor affecting the $B$ chromosome polymorphism is the effect that the $B$ s have on individuals in terms of their physiological and reproductive fitness. When more than $2 \mathrm{Bs}$ are present they lead to a loss in vigour, loss of fertility and reduced genetic activity at the nuclear level (see Jones, 1975, for references). To what extent these harmful effects modify the $B$ frequencies at the population level, through differential selection, is not known either, although it is almost certain that selection for phenotype variation induced by $B \mathrm{~s}$ in rye could only be expected to counter the build up resulting from their accumulation mechanism. There is also the further consideration that the various processes contributing to transmission rates (pairing, chiasma formation, nondisjunction) are themselves sensitive to environmental variation.

In the context of the genetic system as a whole there are benefits which could accrue to future generations through the regulatory effects that $B$ s have over recombination at meiosis. They increase the variance of chiasma frequency of the $\boldsymbol{A}$ chromosomes both between and within pollen mother cells, and this leads to an enhanced level of variability among the progenies of $B$ containing plants. The effect is on the population as a whole, in future generations, rather than on the individual plants which actually carry the $B$ s, and which may be disadvantaged by so doing (Jones, 1975). It is difficult to judge the significance that this kind of effect could make to the equilibrium frequencies of $B$ chromosome populations.

We have taken a fresh look at the dynamics of the $B$ chromosomes in rye, and have attempted to unravel some of the complex interacting factors which contribute to their polymorphism in populations. Our approach has been to identify the main factors affecting their transmission rates and to derive equations representing their behaviour at different stages in the life cycle. These equations have been transcribed into a computer programme which can be used to calculate frequency changes over large rumbers of 
generations in random mating populations. In this way we can evaluate the different parameters which determine their inheritance and find out to what extent their selfish mode of inheritance is in itself responsible for the polymorphism, aside from any effects due to differential fitness and natural selection.

\section{Methods}

The methodology involves the development of a computer model which simulates the inheritance, and change in frequency, of the rye $B$ chromosomes over a large number of generations of random mating. It is not intended that the model should describe the system exactly but that it should simplify and isolate the main factors contributing to the polymorphism in order that we may understand it more easily. The method depends upon a precise characterisation of the mechanism of heredity of the $B \mathrm{~s}$, and a description of the mechanism in simple mathematical terms. The procedure is possible because the $B \mathrm{~s}$ in rye have been thoroughly investigated (Jones, 1975) and we know that their transmission rates from parents to offspring are determined by certain prescribed events occurring at (i) meiosis, (ii) pollen grain and egg cell mitosis, (iii) gametophyte development and fertilisation and, (iv) the sporophyte phase of the life cycle, including effects upon seed fertility. Plants with more than four $B$ s are virtually sterile (Jones and Rees, 1967) and make no contribution to the next generation. In the development of the model therefore we have only taken account of plants within the range $0-4 B \mathrm{~s}$, and any with a greater number than this have been ignored as far as the calculation for $B$-frequencies in the next generation are concerned.

\section{(i) Derivation of equations describing $B$ chromosome transmission}

\section{(a) Meiosis}

Meiosis is complicated by the fact that the $B$ chromosomes are all homologous with one another and we are dealing with individuals that may have $1,2,3$ or $4 B \mathrm{~s}$. In plants with a single $B$ the situation is relatively simple; the $B$ is univalent, it regularly divides at anaphase I, and is included in the telophase nuclei. At the second division it is out of phase in its division cycle and subject to loss by lagging. When the $B$ s are present as a pair their behaviour is at its most regular: they usually form a bivalent at metaphase I and are distributed regularly to the micro- and megaspores at the end of meiosis. With $3 \mathrm{Bs}$ and $4 \mathrm{~B}$ s present in the meiocytes the situation becomes more difficult to describe due to the formation of multivalents as well as bivalents and univalents. For simplicity it is assumed that a trivalent behaves as a bivalent and a univalent, and a quadrivalent as two bivalents. This may not be strictly true, but it is close enough to the real situation for the discrepancies which do occur not to affect the model greatly. Two parameters can thus be seen to determine the transmission characteristics of the $B \mathrm{~s}$ at meiosis: these are $\beta$, the rate of nonpairing when more than $1 B$ is present, and $\lambda$, the rate of loss of the nonpaired univalent $B$ s, which appear as micronuclei at telophase. In deriving formulae to describe $B$ chromosome behaviour at meiosis the terms used 
relate only to the male side, and it is assumed that equivalent processes are occurring with respect to female meiosis. Evidence that this is so comes largely from experiments by Müntzing (1945) and cytological studies by Håkansson (1948). Each $B$ chromosome class of plant is taken separately and the mathematical relationship between the frequencies of each type of microspore produced, and the two parameters $\beta$ and $\lambda$, are determined.

The derivation of equations giving the meiotic output and frequencies of the different kinds of microspores in terms of $\beta$ and $\lambda$, produced by a $2 B$ plant, are shown in fig. 1 and table 1 . Corresponding equations for the
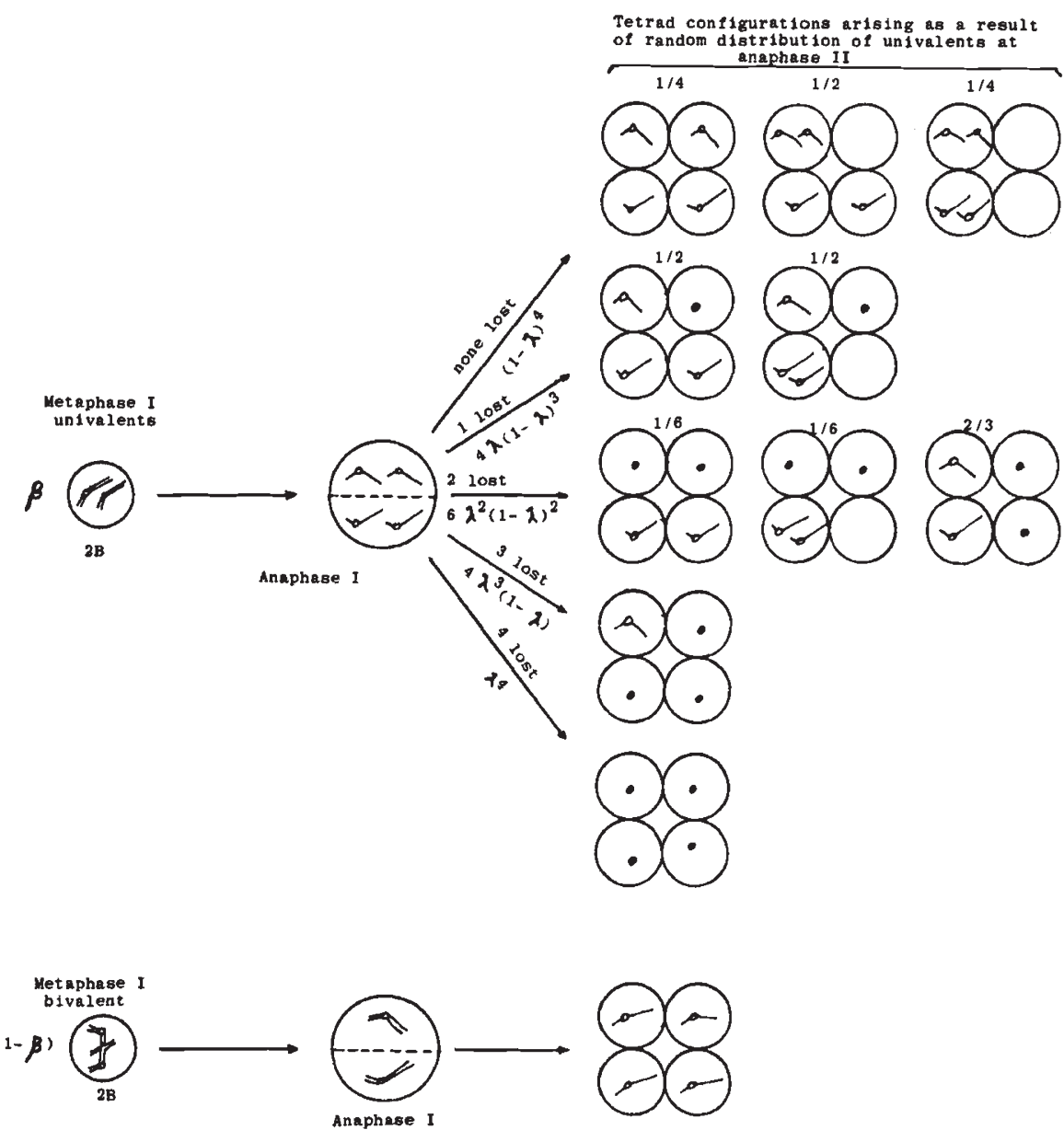

FIG. 1.-Meiotic output of microspores produced by a $2 B$ plant.

meiotic output of $O B$ and $1 B$ plants are relatively simple to obtain, but those for the $3 B$ and $4 B$ classes are more complex and involve many more assumptions and possibilities; the details are given by Matthews (1981) and are presented here only in summary form in table 2.

The parameter $\beta$ can be estimated by observing metaphase $I$ and counting the number of cells in which the $B$ chromosomes are unpaired, 
TABLE 1

Frequencies of the different class of microspores $(O B, 1 B, 2 B)$ produced by a $2 B$ plant, calculated by following each pathway in Fig. 1 back to source and multiplying together individual frequencies contributing to the outcome

$O B: \quad \beta\left[(1-\lambda)^{4}(1 / 8+1 / 8)+\lambda(1-\lambda)^{3}\left(\frac{1}{2}+1\right)+\lambda^{2}(1-\lambda)^{2}(3 / 4+9 / 8+3 / 2)\right.$

1B: $\quad \begin{aligned} & \left.\quad+\lambda^{3}(1-\lambda)+\lambda^{4}\right] \\ & \left.2(1-\lambda)^{4}+2 \lambda(1-\lambda)^{3}+5 / 2 \lambda^{2}(1-\lambda)^{2}+\lambda^{3}(1-\lambda)\right]+(1-\beta)\end{aligned}$

$2 B: \quad \beta\left[1 / 4(1-\lambda)^{4}+\frac{1}{2} \lambda(1-\lambda)^{3}+1 / 4 \lambda^{2}(1-\lambda)^{2}\right]$

$: 1 / 4 \beta(1+\lambda)^{2}$ $: 1-\frac{1}{2} \beta\left(1+\lambda^{2}\right)$

and $\lambda$ can be estimated from counting the number of micronuclei present in tetrad microspores, working on the assumption that each micronucleus represents $1 B$.

\section{(b) Pollen grain and egg cell mitosis}

Experimental matings, as well as direct cytological observations, have shown that the $\boldsymbol{B}$ chromosomes are capable of undergoing chromatid nondisjunction at the first pollen grain mitosis, and that the $B$ s are frequently directed into the generative nucleus in an unreduced number (Müntzing, 1946). Equivalent behaviour happens at first mitosis of the megaspore, in the female gametophyte (Håkansson, 1948). On both the male and female side, however, there is a certain amount of infidelity in both the nondisjunction event itself, and in the direction in which the nondisjoined chromatids move. When more than $1 B$ is present in the developing gametophyte there are of course more complex combinations of events to be taken into account, but notwithstanding this problem two parameters can again be used to describe the behaviour of the $B$ s at this stage, and these are $\mu$, the rate of nondisjunction and $\alpha$, which denotes the proportion of the nondisjoined chromatids included in the germ linetermed the direction rate. It is assumed that nondisjunction and direction are two independent processes. A microspore carrying $2 B$ chromosomes is used as an example (fig. 2 and table 3 ) to show how equations are derived which give the frequencies of the different classes of gametes produced, in terms of $\mu$ and $\alpha$. As with meiosis the derivation of these equations is less and more complex, respectively, for microspores with lower and higher numbers of $B \mathrm{~s}$. A summary of the equations which give the frequencies of different types of gametes, produced by the five classes of microspores concerned is given in table 4, and the full details of their derivation can be found in Matthews, (1981).

\section{(c) Male gametophyte development}

$B$ chromosomes affect the physiology and competitive ability of the pollen grains which carry them, and although it is known that in certain cases, i.e., $2 B$ pollen, the $B$ s may actually promote higher germination and faster pollen tube growth (Puertas and Lacadena, 1974; Puertas and Carmona, 1976), we have taken the simple view that in general $B$ s are 


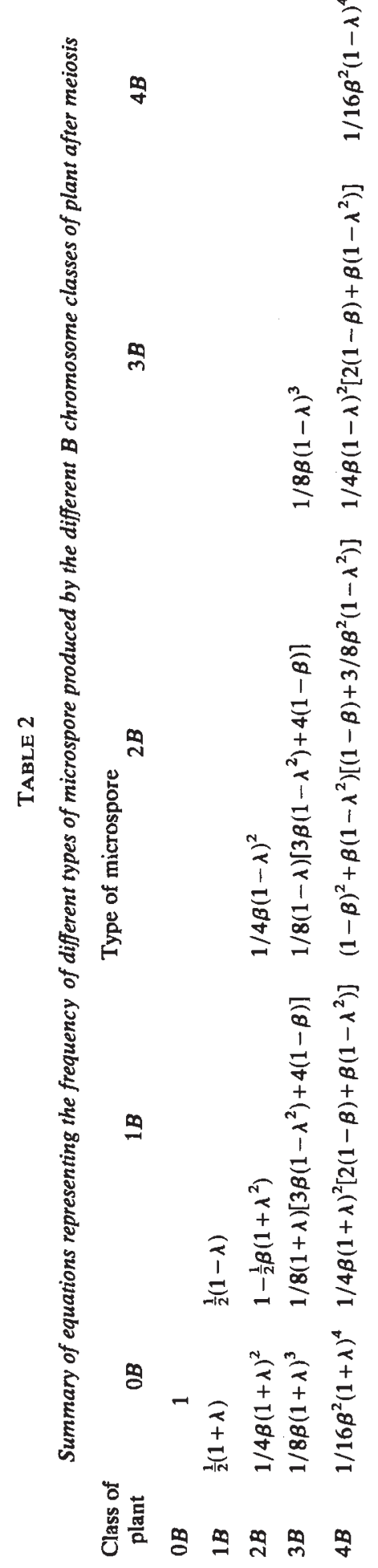




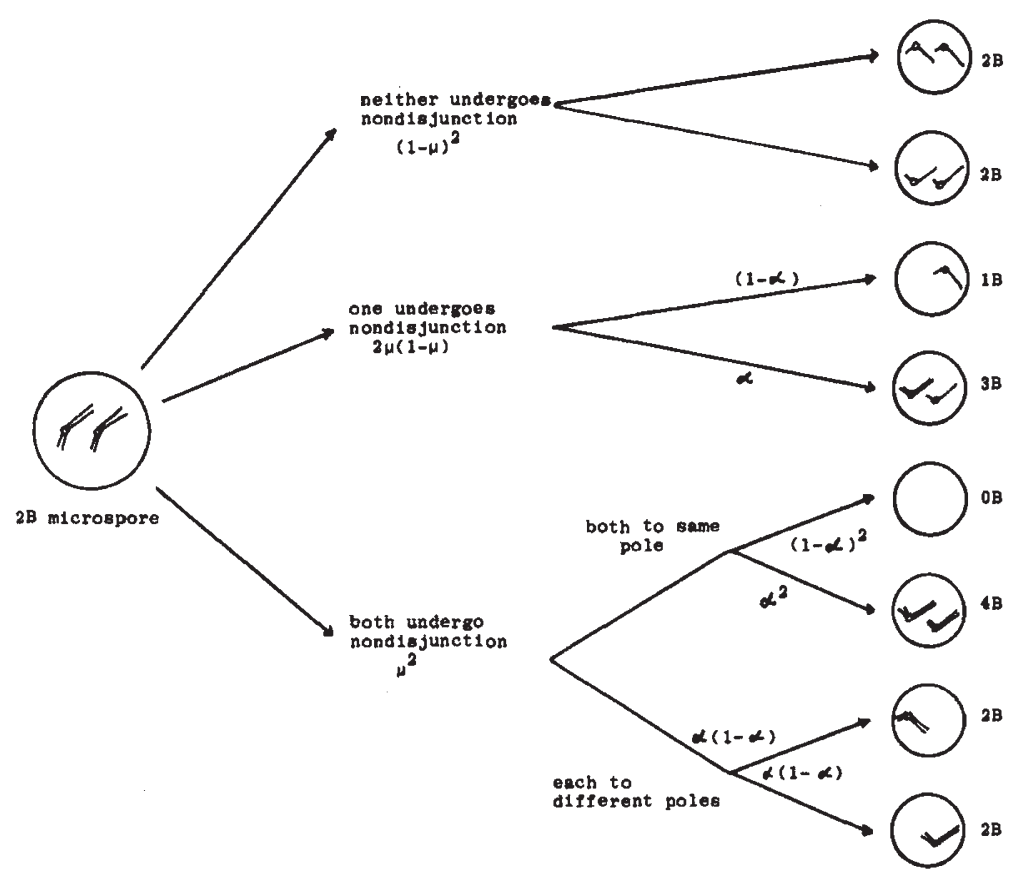

FIG. 2.-Possible types of gametes produced from a $2 B$ microspore.

detrimental to cell physiology and that increasing their numbers will lead to a progressive impairment of pollen grain function. Weighting factors have therefore been introduced to take into account the variation in fitness levels of the different $B$-classes of male gametophyte (no corresponding adjustments have been made on the female side). The effect has been described using a parameter called the gametic selection gradient, $g$, which is a measure of the gradient of a line on a graph that begins at 1.0 , where the $B$-class is zero, and passes through to a value of $1-g$ where the $B$-class is 4 . Thus the equation for calculating the gametic weighting factors for each $B$-class is given by $1-\frac{1}{4} B g$, where $B$ is the value of the $B$-class $(0,1,2,3,4)$ and $g$ the value of the selection parameter. The weighting system is only approximate and takes no account of any odd-even effect (Jones and Rees, 1969) or of the stimulatory effects found for $2 B$ pollen.

\section{TABLE 3}

Frequencies of each class of gamete produced by a $2 B$ microspore, found by multiplying together the frequencies in each pathway of the diagram in fig. 2

\begin{tabular}{cl} 
Gamete $B$-class & \multicolumn{1}{c}{ Frequency } \\
$O B$ & $\mu^{2}(1-\alpha)^{2}$ \\
$1 B$ & $2 \mu(1-\mu)(1-\alpha)$ \\
$2 B$ & $2 \mu^{2} \alpha(1-\alpha)+(1-\mu)^{2}$ \\
$3 B$ & $2 \mu(1-\mu) \alpha$ \\
$4 B$ & $\mu^{2} \alpha^{2}$
\end{tabular}




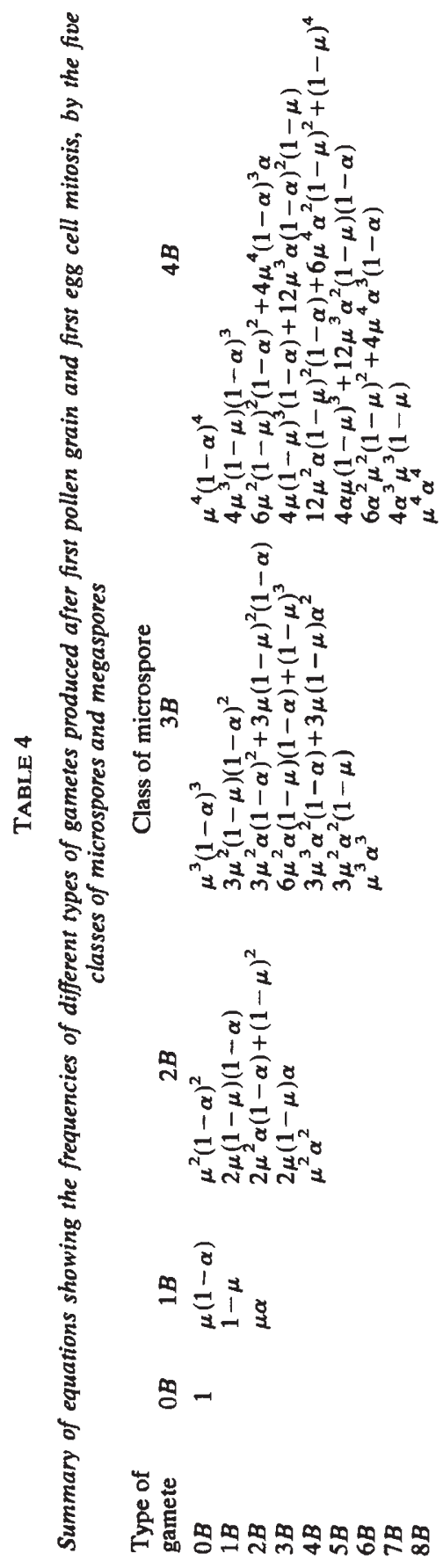


It does, however, facilitate the study of gametic selection effects by using a single parameter which can be tested at various values while other parameters are held at a constant level.

\section{(d) Sporophyte phase of the life cycle}

It is well established that plants with differing numbers of $B$ s also have differing, and usually inferior, competitive abilities. Plant weighting factors, similar to the gametic weighting factors, have been introduced to make some allowance for this variation in physiological and reproductive fitness.

\section{(ii) The computer model}

The equations that have been derived for describing the behaviour of the $B \mathrm{~s}$ at four stages of the lifecycle form the basis of the model for calculating changes in $B$-frequency, and for elucidating the principal factors involved in determining the equilibrium level of $B$ chromosomes in populations. A computer programme was developed so that the many calculations required over a large number of generations could be performed quickly and accurately. The resulting changes in $B$ chromosome frequency were then displayed using a graph plotter linked to the computer. Provision is made in the programme for one parameter at a time to be varied between certain limits, while the others are held at constant specified values, so that the effect of altering that one parameter can be investigated (Matthews, 1981).

\section{Results}

The computer simulation programme can obviously be manipulated to generate numerous graphs displaying changes in $B$ chromosome frequency. It is possible to consider the consequences of setting the initial $B$-levels, and the individual parameters, at many different values, as well as investigating the effects produced by different combinations of parameter values. We have avoided as many of these diversions as possible, and have concentrated on presenting some simple graphs obtained by varying one parameter at a time, at relatively few values, and this has enabled us to identify and evaluate the contribution that each parameter makes to determining the $B$-frequencies in populations. For the purposes of presenting the graphs the average $B$-level per plant is defined as the total number of $B$ chromosomes in the population divided by the total number of plants, and can be expressed as $=B_{1}+2 B_{2}+3 B_{3}+4 B_{4}$, where $B_{1}, B_{2}, B_{3}$ and $B_{4}$ are the frequencies in the population of $1 B, 2 B, 3 B$ and $4 B$ plants respectively. As the model assumes that plants with more than $4 B$ chromosomes are inviable or sterile, then obviously the maximum average $B$-level that can be attained will be $4.0 B$ s per plant, when all the individuals in the population are $4 B$ plants.

In fig. 3 is shown the effect of different levels of nonpairing of the $B \mathrm{~s}$, from 0.0 (all paired) to 1.0 (all univalent), when the nonpaired loss rate is set at 0.30 and the other parameters are all held at their maximum positive values. A nonpaired loss rate of 0.30 , rather than 0.00 (no loss), was used for this graph because it gives a wider separation of the lines and 
makes for a clearer appreciation of the effect due to variation in nonpairing rates. In the simulation shown in fig. 3 , as in most of those that follow, the initial $B$-level is set at a low value of $0.01 B /$ plant, and the graph shows the build up of $B$ s over an arbitrary 65 generations of random mating. The accumulation of the $B \mathrm{~s}$ is rapid, and it is interesting to note that it takes place even with a non-pairing rate of $1 \cdot 0$, i.e., when all of the $B \mathrm{~s}$ are univalent. The build up proceeds even faster, and results in higher equilibrium levels, when the nonpaired loss rate is given values lower than $0 \cdot 3$.

Clearly there are numerous interrelationships that could be considered between the different parameters, but the main outcome is that the model has identified that the effect of different rates of nonpairing of the $B \mathrm{~s}$ is to generate different equilibria in populations, corresponding to the different rates of elimination of the $B$ s at meiosis.

Estimates of values of the nonpairing rate $(\beta)$, together with those for the other parameters, will be discussed in some detail in a subsequent publication in this series. Suffice it to say for the time being, that the mean estimated value for nonpairing in rye is of the order of $0 \cdot 2$, and the maximum 0.7 . The values used in the graph in fig. 3 (below 0.7 ) are therefore quite realistic and well within the range that may be encountered in natural or experimental situations.

Variations in the nonpaired loss rate parameter $(\lambda)$, given a nonpairing rate of 0.50 and maximum positive values for the other parameters, produces a virtually identical outcome, as is shown in fig. 4 . The effect is again to regulate the final mean equilibrium $B$-level per plant, and the number of generations taken for the equilibrium levels to be attained. The maximum equilibrium level of $4 B$ /plant is not reached because the nonpairing rate has been arbitrarily set at a value of $0 \cdot 50$. We note also, the remarkable fact that even with a nonpairing rate of 0.50 , the $B$ s are still capable of accumulating in the population in the face of a nonpaired loss rate of as high as 90 per cent, provided of course that directed nondisjunction is operative. If minimum values are given for nondisjunction and direction rates, and the nonpairing rate is again set at $0 \cdot 50$, the $B$-level quickly falls from an initial mean of $2 \mathrm{~B} /$ plant down to zero, if there is any degree of nonpaired loss rate. Estimates of the nonpaired loss rate parameter $\lambda$, made from the literature, gives a mean value of 0.34 and a range from 0.00 up to 0.86 (Matthews and Jones, in preparation).

Thus it is evident from the results displayed in figs. 3 and 4 that variation in both nonpairing and nonpaired loss rate can provide a means for adjusting the level of $B$-frequencies in populations.

In fig. 5 is shown the consequences of varying the nondisjunction rate, given maximum values for all other parameters. The outcome contrasts with that for meiotic elimination, in that the differing rates of nondisjunction have much less effect upon the final equilibrium $B$-level/plant and relatively more upon the rate of build-up of $B \mathrm{~s}$. The final equilibrium frequencies rise very high and are reached very quickly, over a wide range of values for the nondisjunction parameter $\mu$. If the direction rate parameter $\alpha$ is set at 0.75 , instead of 1.00 , a similar result follows, but it is modified in that the population never reaches the maximum level of $4 B /$ plant. The mean estimated value of $\mu$, from the literature (Matthews and Jones, in 


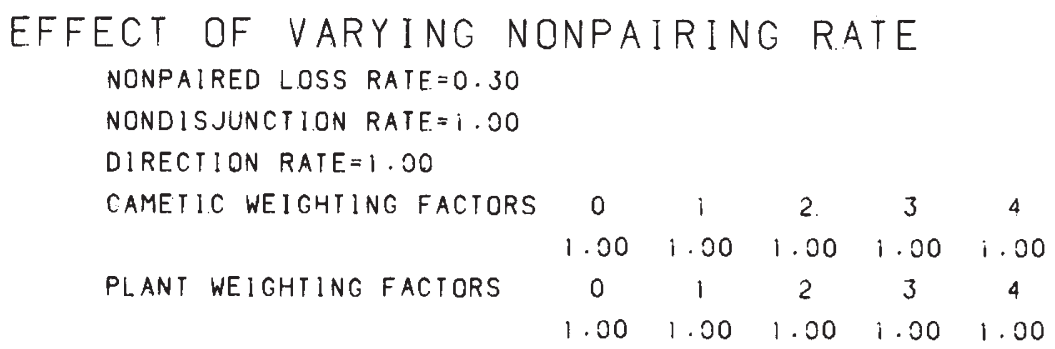

INITIAL. B LEVEL $=0.01$

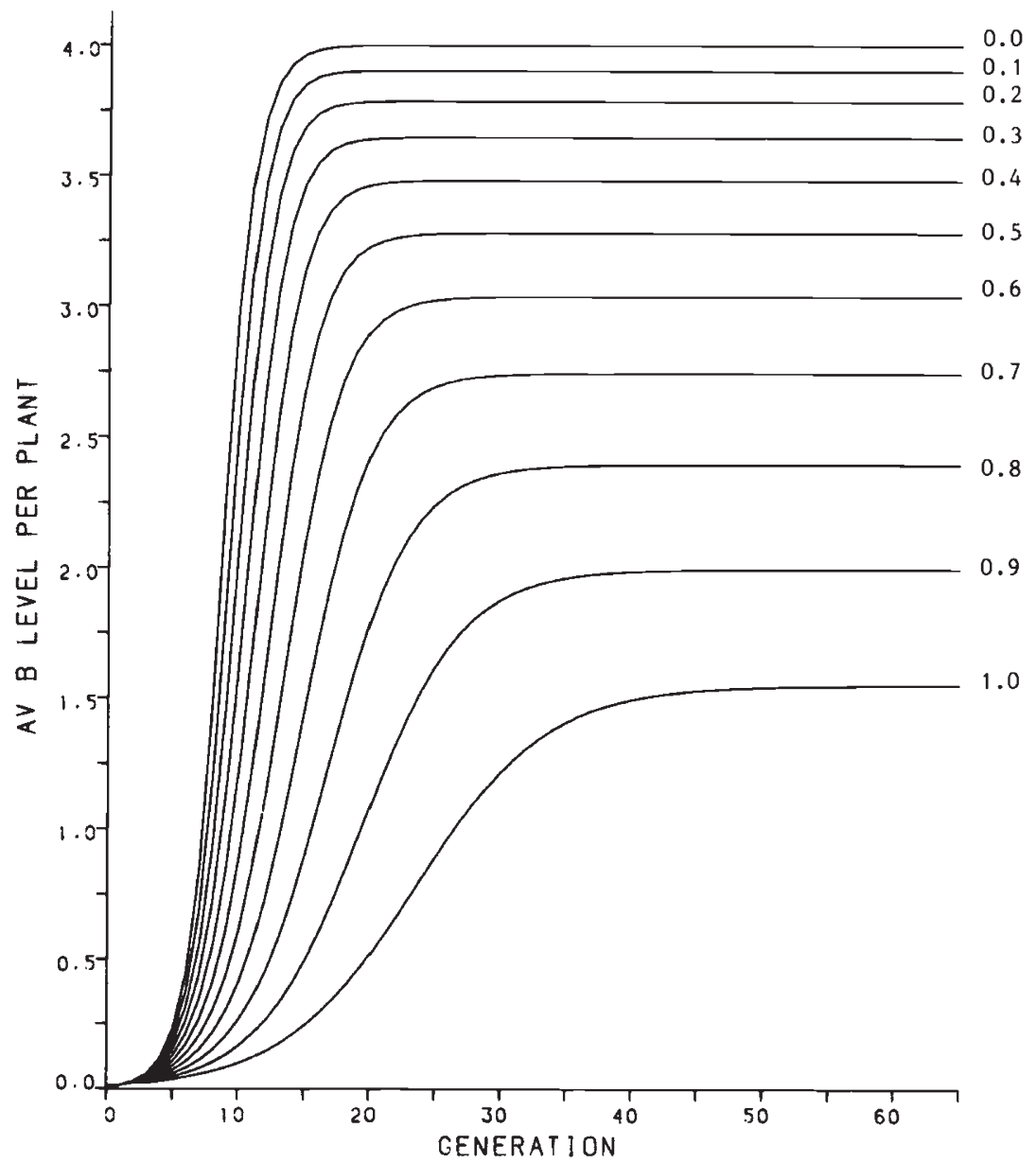

FIGS. 3-9.-Computer generated simulation graphs showing how parameters controlling the transmission of $B$ chromosomes in rye affect equilibrium frequencies in populations. 


\section{EFFECT OF VARYING NONPAIRED LOSS RATE NONPAIRING RATE $=0.50$ \\ NONDIS JUNCTION RATE $=1.00$ \\ DIRECTION RATE $=1.00$

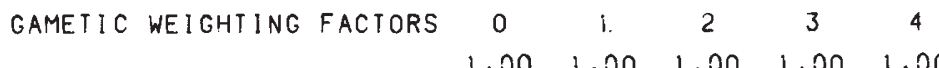 \\ PLANT WEIGHTING FACTORS 001124 \\ INITIAL B LEVEL $=0.01$

$\begin{array}{lllll}1.00 & 1.00 & 1.00 & 1.00 & 1.00\end{array}$

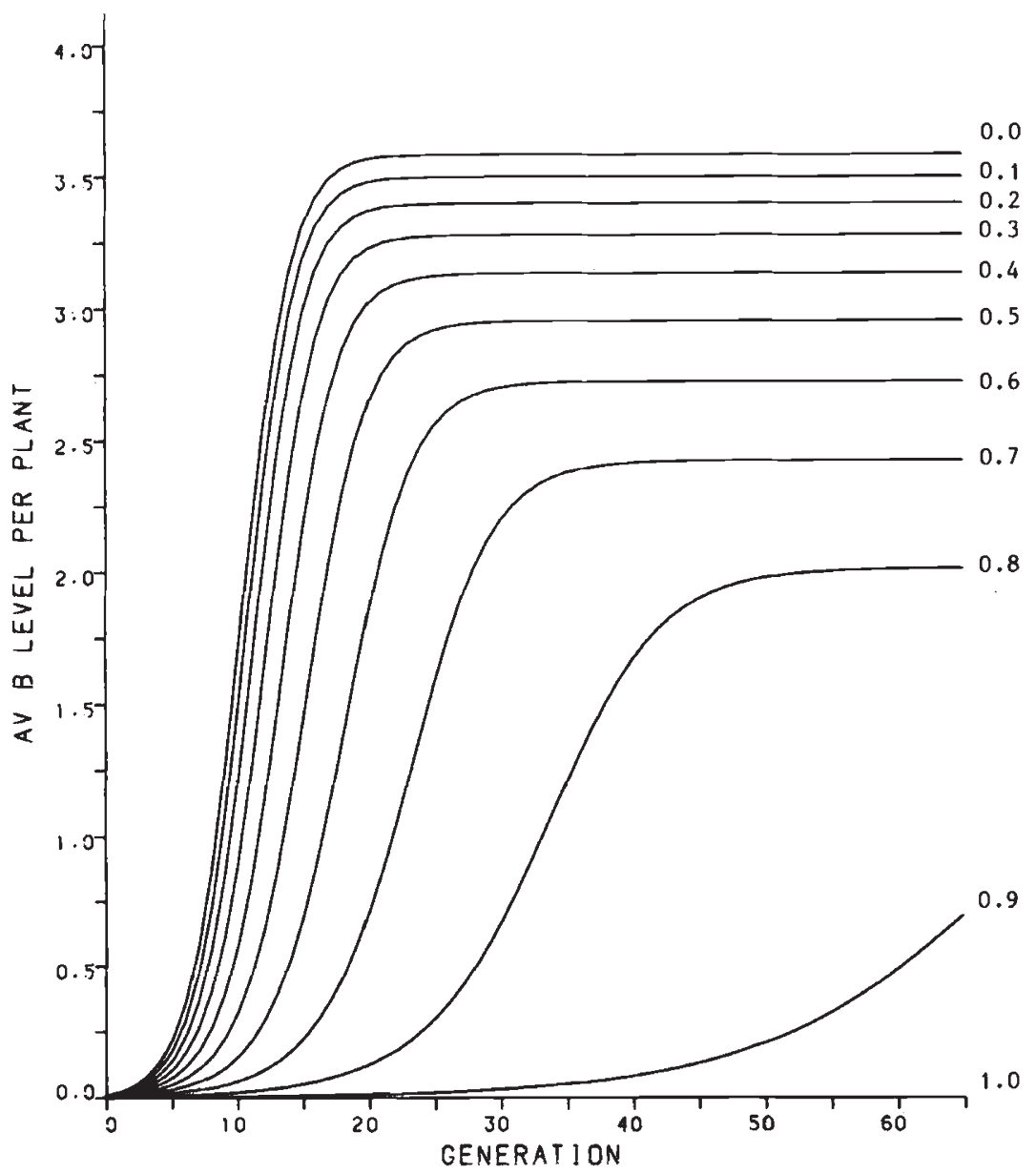

FIG. 4. 

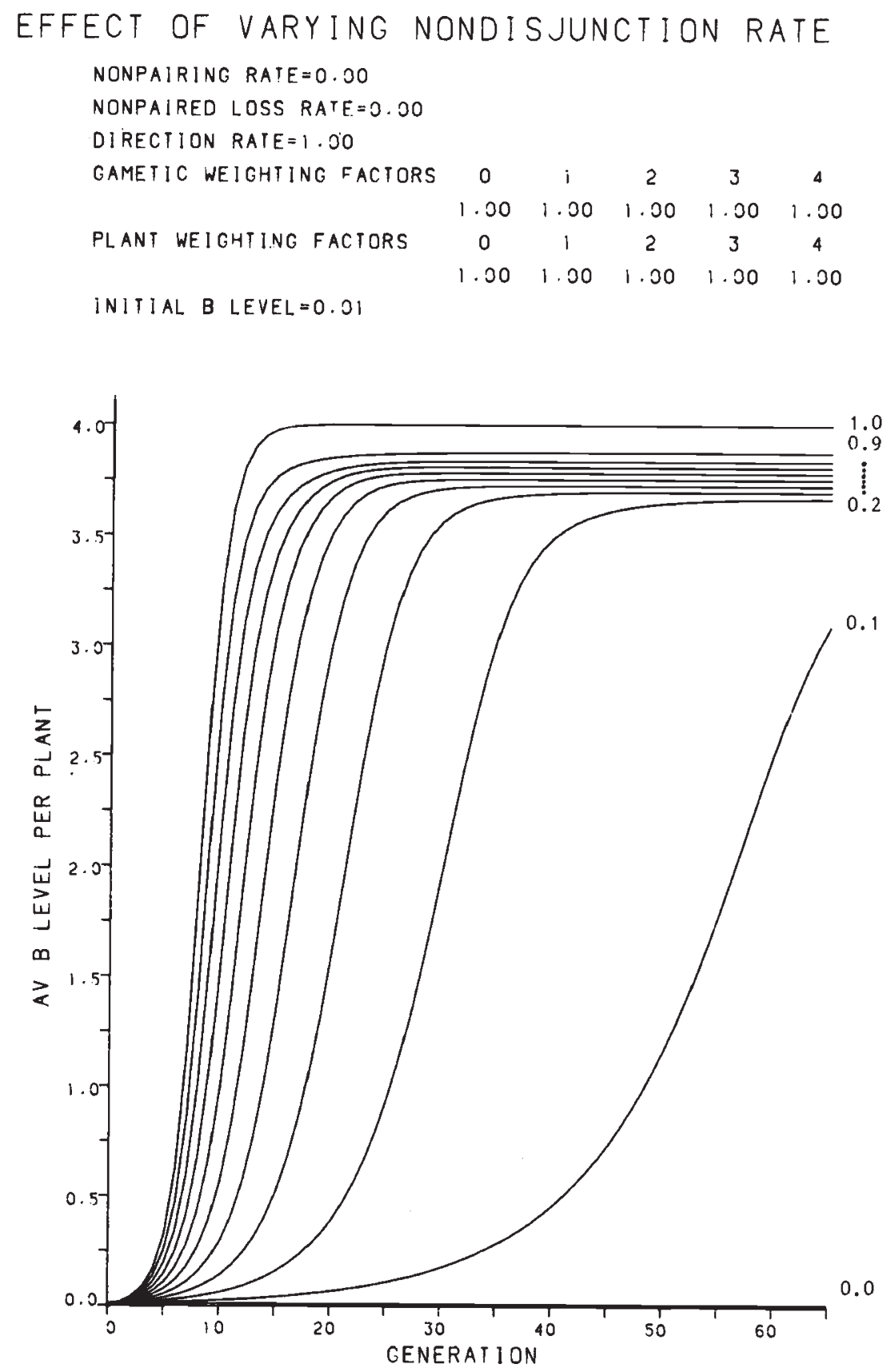

FIG. 5. 


\section{EFFECT OF VARYING DIRECTION RATE}

NONPAIRING RATE $=0.00$

NONPAIRED LOSS RATE $=0.00$

NONDISJUNCTION RATE $=1.00$

GAMETIC WEIGHIING FACFORS $0 \quad$ i $2 \begin{array}{lll}2 & 3\end{array}$

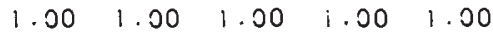

$\begin{array}{lcccccc}\text { PLANT WEIGHTING FACIORS } & 0 & 1 & 2 & 3 & 4 \\ & 1.00 & 1.00 & 1.00 & 1.00 & 1.00\end{array}$

INITIAL B LEVEL $=0.0 i$

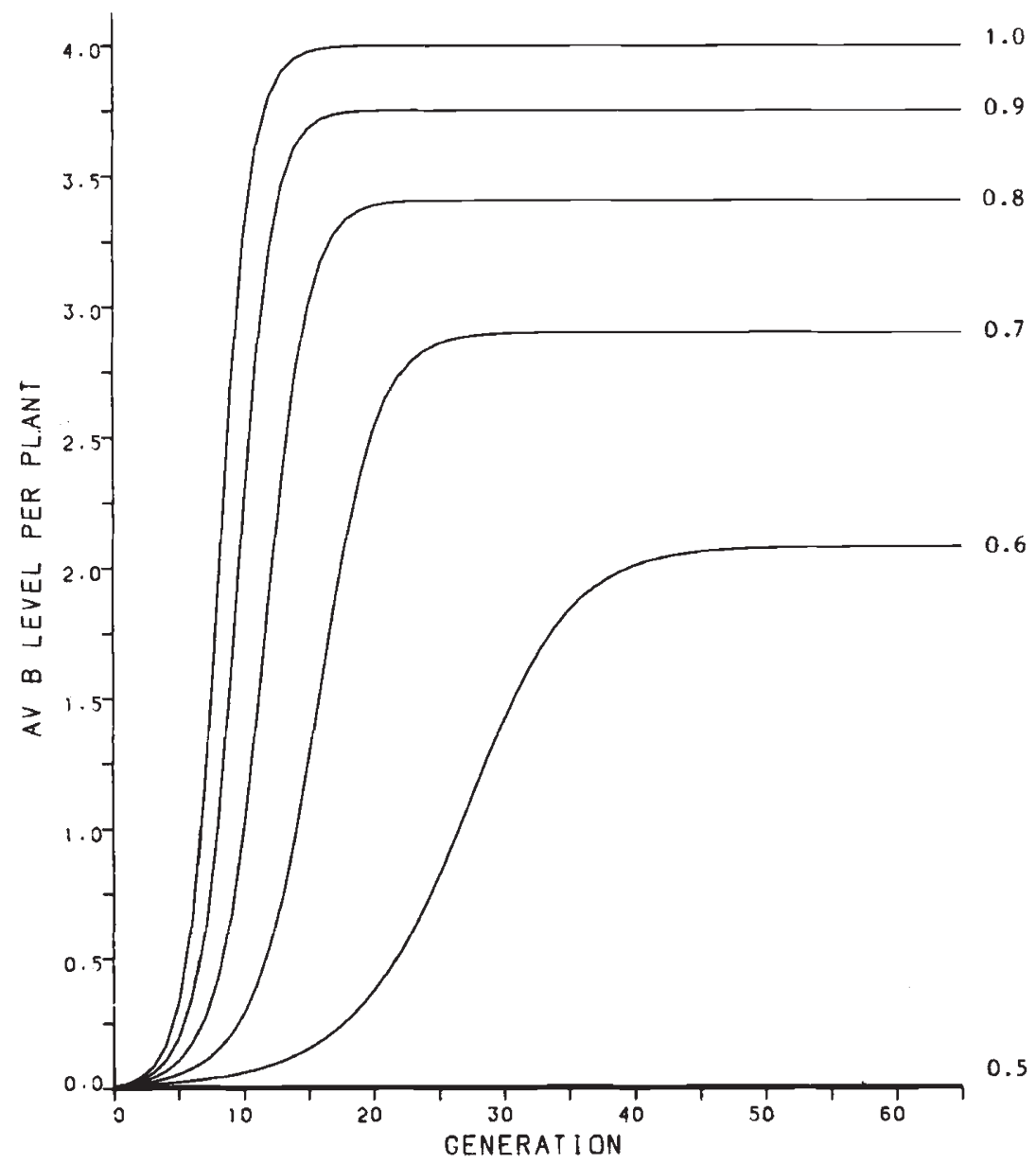

FIG. 6. 
EFFECT OF VARYING DIRECTION RATE

NONPAIRING RATE $=0.00$

NONPAIRED LOSS RATE $=0.00$

NONDIS JUNCTION RATE $=1.00$

GAMETIC WEIGHTING FACTORS $0 \quad$ i 23034

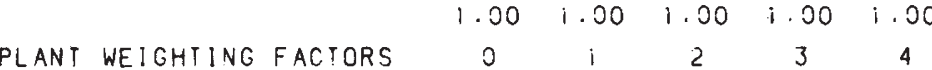

$1.00 \quad 1.00 \quad$ i.00 $1.00 \quad 1.00$

INITIAL B LEVEL $=2.00$

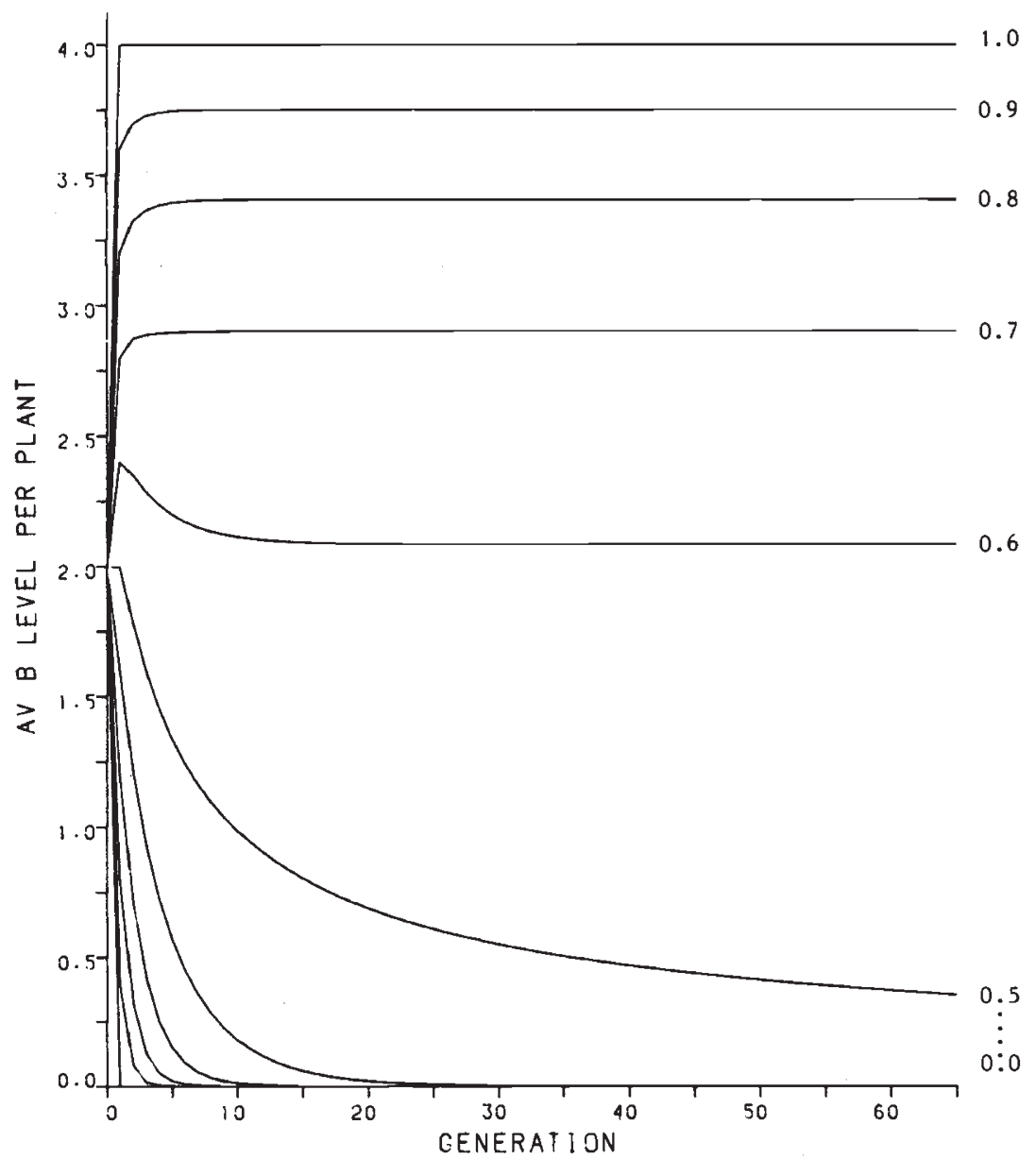

FIG. 7. 


\section{EFFECT OF VARYING GAMETIC WEIGHTING \\ FACTORS}

NONPAIRING RATE $=0.00$

NONPAIRED LOSS RATE $=0.00$

NONDIS JUNC !ION RATE $=0.90$

DIRECTION RATE. $=0.90$

PLANT WEIGHIING FACTORS $0 \quad 1 \quad 2 \quad 34$

INITIAL.B LEVE.L $=0.01$

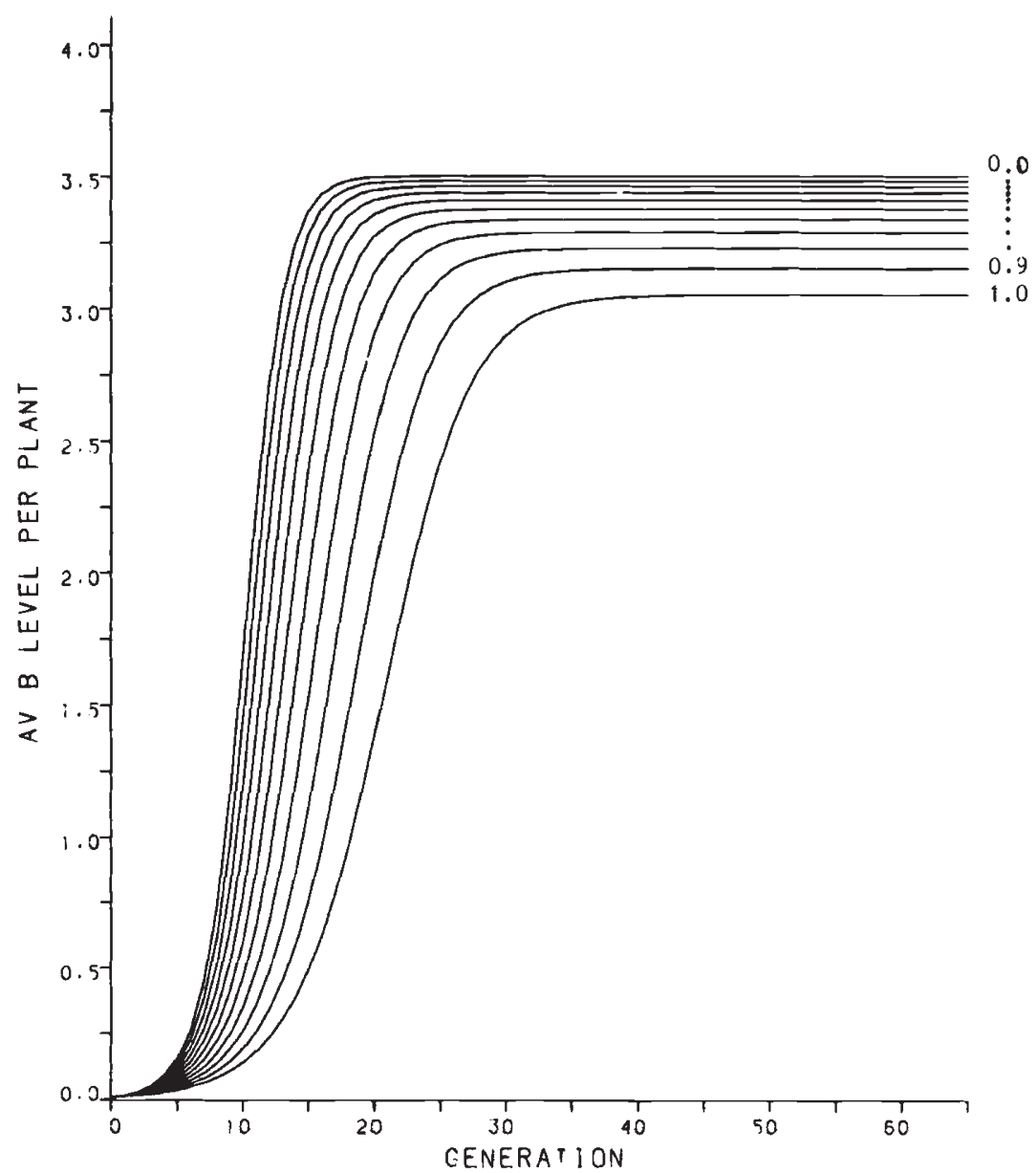

FIO. 8. 


\section{EFFECT OF VARYING PLANT WEIGHTING \\ FACTORS}
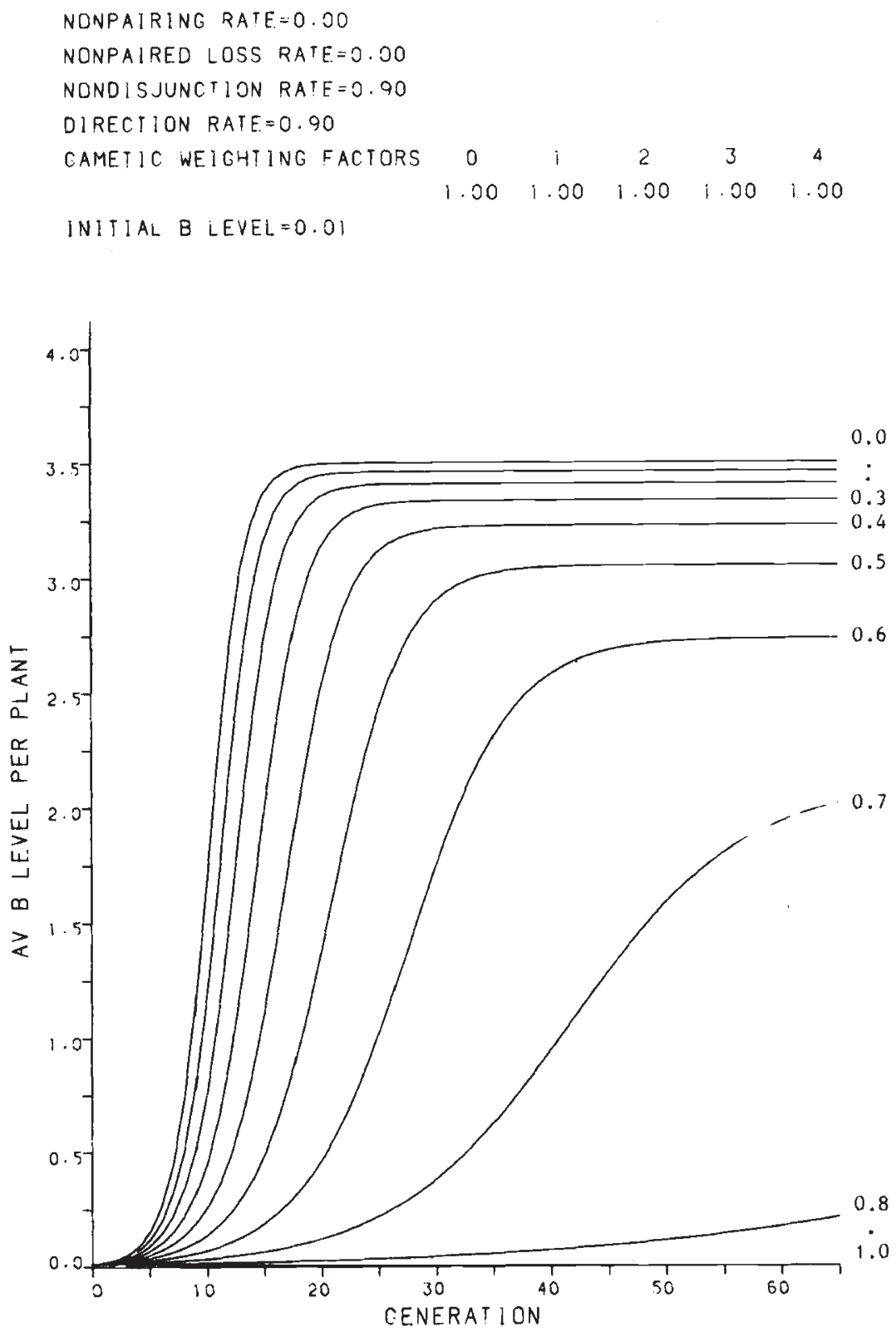

FIG. 9. 
preparation), is of the order of 0.86 and the range is narrow, from 0.76 0.95 .

The consequences to the polymorphism of varying the direction rate (i.e., whether the nondisjoined $B$ chromatids pass into the generative or the vegetative nucleus at first pollen grain mitosis) have hitherto received scant consideration. The results of the simulation for changes in this parameter are shown in fig. 6 , under conditions where the other parameters are held at maximum positive values. The outcome is as expected, in so far as the $B$ s are only able to accumulate when they nondisjoin to the generative pole with a frequency greater than $0 \cdot 5$. Values for the direction rate inbetween 0.5 and 1.0 give the appropriate range of equilibrium $B$-levels between 0 and 100 per cent. A similar graph is produced even when the nondisjunction rate is set as low as 0.5 . With an initial $B$-level of $2 B /$ plant the frequency of $B \mathrm{~s}$ in the population declines to complete loss with a direction rate parameter $\alpha$ of 0.5 or less (fig. 7). Direction rate is therefore a highly significant parameter in regulating the equilibrium $B$-frequency level in rye populations, and it is essential for the survival of $B \mathrm{~s}$ in the first place to have a value of $\alpha$ which is in excess of 0.5 . Estimates from the literature suggest a mean parameter value for rye of 0.85 and a range of values from $0.65-1.00$ (Matthews and Jones, in preparation).

The effect of varying the gametic weighting factors, by introducing a bias in fitness against the male gametophytes is shown in fig. 8. As explained earlier, equivalent levels of reproductive and physiological fitness are assumed for all the $B$ classes of gamete on the female side. In the simulation the parameters for nondisjunction and direction rate have been set at realistic values of 0.90 , and all the other parameters at their maximum positive values. The outcome is surprising: over the full range of gametic weighting parameter variation from $0 \cdot 0-1 \cdot 0$ there is only a small drop of some 15 per cent in equilibrium level, and a slight delay in the number of generations taken to attain equilibrium. It is possible therefore, with the gametic weighting factor set at $1 \cdot 0$, to remove all the $4 B$ male gametes (with correspondingly reduced levels of bias against the $3 B, 2 B$ and $1 B$ classes) at each generation, and to have remarkably little effect upon the polymorphism. Such high levels of impaired gamete fitness are unrealistic of course, and although we have not obtained any reliable estimates for normal values of this parameter there is no reason to believe that any such large bias against $B$ containing gametes is ever found in nature. On the contrary, and as already mentioned, there is some evidence to suggest that the competitiveness of gametes can actually be enhanced by the presence of low numbers of $B$ s. In any event the system, if necessary, could cope easily with heavy selection against $B$ s at this stage of the life cycle. The final equilibrium level which is reached naturally depends upon the values given to the other parameters as well, but when viewed in isolation, as the graph in fig. 8 clearly shows, the effect of gamete selection is relatively small and over the range of variation found in nature is not likely to have much impact upon the population dynamics of the $B$ chromosome system in rye. If the nondisjunction and direction rate parameters are given their maximum values of 1.0 , then the build up of the $B$ s proceeds to the maximum of $4 B$ /plant and the selection against them is quickly and completely overcome. 
Selection at the plant level has a more significant and severe effect upon the equilibrium levels reached, and as fig. 9 shows selection at this stage of the life cycle is a very effective way of adjusting the equilibrium levels in populations. In the graph shown in fig. 9 the nondisjunction and direction rate parameters are again given levels of 0.9 , and as before variation in both of these parameters will modify response to the plant weighting factors themselves. In reality this parameter is expected to be an important one because of the severe effects of $B$ s upon seed set, which is the main factor concerned. Estimates from the literature (Müntzing, 1943) suggest values within the range 55-77 per cent selection against $4 B$ plants, with corespondingly smaller levels for the other classes. As the graph shows, however, the build up of $B$ s will proceed even with a plant weighting factor of 0.8 . At the lower end of the range, up to the level where 50 per cent of the $4 B$ plants are being eliminated, the effect of loss of the $B$-containing individuals is relatively minimal, and easily countered provided of course that nondisjunction and direction rate are operating at high levels. In summary, it would appear that the elimination of $B$ s by plant selection is a sensitive mechanism for adjusting equilibrium $B$ levels, but that high levels of elimination can be tolerated without any likelihood of the polymorphism itself being run down.

\section{Discussion}

The model described here is to some extent a statement of the obvious; namely that the $B$ chromosomes in rye can increase in number and spread throughout a population, once they have arisen or have been newly introduced. It asserts also that not only is the mechanism of inheritance effective in accumulating $B \mathrm{~s}$, but that it can by its own nature, and potency, account entirely for the observed $B$ chromosome polymorphism in natural populations, without any recourse to natural selection. The system can generate and develop a widespread polymorphism even in the face of heavy selection against gametes and plants that carry the $B$ chromosomes. It can do this because the inbuilt mechanism of directed nondisjunction, with which the $B$ s are endowed, is operative at such a high level of effectiveness, that it can counter the negating effects that arise from loss at meiosis and by reduced levels of seed fertility. Any positive selective advantage at either the gamete or plant level will simply provide a further boost to the rate at which the $B$ s increase in frequency.

The impetus for this work arose in the first instance from observations by the authors on a small open pollinating population (ca. 600 plants) of $B$ chromosome rye originating from Korea. At the initial planting the frequency of $B$ s in the seed sample (mostly the $2 B$ class) was of the order of two per cent. Within the five generations for which the population was monitored this had risen to more than 40 per cent, and led to the feeling that such a dramatic rate of increase was possibly inevitable, and quite independent of any superior (or otherwise) form of fitness conferred upon the individuals which carried the $B \mathrm{~s}$. The results of the computer simulation, taken together with our experience and knowledge of the transmission characteristics and phenotypic effects of the $B$ s in rye, now confirm and substantiate this feeling. The only way in which a "fit" $B$ chromosome can fail to survive, and fail to selfishly propagate itself, is by having unusual, 
and consistently low, values for the parameters to which we have referred. We will consider the question of estimates for, and variation in, the values to be ascribed to these parameters, in a subsequent paper. The question is quite an involved one, but as far as we can see, and as we have already suggested, the values of the parameters concerned are naturally found at extremely high levels and are fully consistent with the claims that the spread and accumulation of the $B$ s, albeit to varying degrees, is inevitable.

In the simulations that have been described the starting points have been set at an arbitrary, and low, level of $0.01 \mathrm{~B} /$ plant. It is appreciated that it could take many generations to arrive at even this low frequency, where the initial origin of the $B \mathrm{~s}$ in a population is confined to only one plant. Nothing is known about the origin of $B \mathrm{~s}$ in rye, and we can say little about the nature of the early stages of their establishment. The only statement that can be made with certainty is that if a new $B$ arises, complete with the vital property of directed nondisjunction, then it will only have to survive the rigours of passing through one cycle of meiosis before it is provided with a homologue. From then on its inheritance is secure.

Another aspect of the polymorphism which we have not considered in any detail, and one which may impose its own constraints and limitations upon the output of the model, is the question of population size. We have made the assumption that mating takes place completely at random and that every male gamete has potential access to every female one. This condition would only apply where the initial distribution of $B$ s was thinly and widely spread and the absence of this circumstance could hinder the accumulation processes in the very early stages of establishment. The accumulation could be modified as well by the fact that there is a certain degree of assortative mating in $B$ chromosome rye. Plants with $B$ s have a slower rate of development and come into anthesis later than those without (Jones and Rees, 1968).

One further question which arises, as a result of the findings of this model, is how widely distributed are the $B$ chromosomes in rye, and why is it that we do not find them at levels of 100 per cent frequency in all populations? The latter part of the question is the most easily dealt with because it has already been answered by reference to estimates of parameter values taken from the literature: the parameters which determine the $B$-frequency are high, but are not at their maximum positive values in natural situations. On the question of distribution we are only really concerned with "natural" or "primitive" strains of rye, and not with the more highly selected cultivated varieties from which $B$ chromosomes are virtually absent. The highest $B$-frequencies that have been recorded are over 90 per cent in a "native" Japanese rye population (Kishikawa, 1965). In populations from Korea, Lee and Min $(1965 a, b)$ found $B$ s in all 32 of the populations that they investigated, in frequencies ranging from 2-73 per cent. In a separate study Müntzing $(1957 a, b)$ found them in all seven of his Korean populations and they ranged in frequency from 19-90 per cent. The other major survey was done in Yugoslavian rye (Zecěvić and Paunović, 1967) where all 25 populations sampled had $B$ s, and where they ranged in frequency from 10-55 per cent. The only surveys reporting on the absence of $B$ s in some population samples, are for primitive rye strains from Iran (Kranz, 1963) and Afghanistan (Müntzing, 1950). In none of the populations mentioned above do we have sequential samplings over 
several seasons, and in none of them do we know whether or not the $B \mathrm{~s}$ have reached their equilibrium frequencies. What is important, however, and what is fully at one with the model, is that natural populations of rye do exist with $B$-frequencies of up to 90 per cent or more, and in these populations there is no evident explanation for the existence of these $B \mathrm{~s}$ other than that they have the facility to increase in number in the germ line, in comparison to the $A$ chromosomes of the basic complement.

The model that we have developed here could readily be adapted to cover many variations in the transmission characteristics of $B \mathrm{~s}$, as found in other species. We have in fact run the programme assuming the more common situation of directed nondisjunction on the male side only, and found a similar result: the main difference being one of time scale for the accumulation. We have not attempted to test any other variations.

It is important to emphasise too that because the $B$ chromosome polymorphism in rye and other species can be generated and maintained, and accounted for, through the agency of an accumulation mechanism, that this mechanism is not necessarily the only means by which the $B$ s may survive and be regulated in populations. Species are known where there is no apparent mechanism of increase, and no obvious favourable phenotypic effects either, yet the $B$ s still exist (or are continually arising?) at high frequencies within their populations, e.g., Allium cernuum (Grun, 1959) and Allium schoenoprasum (Bougourd and Parker, 1979). There are other well known cases too, where the distribution pattern of the $B$ s is closely correlated with variation in one or more components of the environment, such as humidity (Centaurea scabiosa, Fröst, 1958); soil composition (Festuca pratensis, and Phleum phleoides, Bosemark, 1956, 1967); temperature and rainfall (Myrmeleotettix maculatus, Hewitt and John, 1967, 1970; Hewitt and Ruscoe, 1971); and in all of these cases it is usual to find the $B \mathrm{~s}$ in their highest frequencies under conditions which are most favourable to the species concerned. Precisely how selection is operating in these cases is not known. It could be effective directly by acting upon the differential fitness of individuals with and without $B \mathbf{s}$, or indirectly by influencing the transmission process of the $B$ s themselves. There is evidence from experimental studies for both kinds of effect. Experiments in rye and perennial ryegrass, in which plants have been grown under extreme conditions of density stress, have demonstrated that selection may discriminate both against (rye) and in favour of (ryegrass) the $B$-containing individuals (Rees and Hutchinson, 1973). The situation in ryegrass is unique in that it is the only case known where the $B$ s have been shown to confer a selective advantage upon the individuals which carry them, albeit under experimental conditions (Hutchinson, 1975). Studies by Kishikawa (1970), again in rye, have shown that the transmission rates of the $B$ s can vary in different environments. We have also carried out experiments under controlled growth conditions which demonstrate how differences in environment can modify the transmission and accumulation of $B \mathrm{~s}$ in rye, and these results will be described in a separate publication.

The idea that $B$ chromosomes may be more useful to themselves than they are to the organism that carries them is not a new one. It was first proposed in a discussion paper by Östergren in 1945, in relation to the $B$ s in rye, and has more recently received strong support from Kimura and Kayano (1961), working with Lilium callosum, and from Nur's studies on 
the mealy bug, Pseudococcus obscurus (Nur, 1966, 1969) and the grasshopper Melanoplus femur-rubrum (Nur, 1977). Nur has shown that in both of these species the $B$ s are harmful to the fitness of the individuals that carry them, and are maintained at equilibrium frequencies in populations only by virtue of their respective accumulation mechanisms. There is evidence for selfishness too from crossing experiments and from population surveys in Myrmeleotettix maculatus (Hewitt, 1973a, b, 1976), although the data are more equivocal than that for the three species mentioned above. Mechanisms of "drive" which lead to $B$ chromosome accumulation have been described in numerous species of both plants and animals, and they are principally of three kinds.

(a) Premeiotic drive is based on mitotic nondisjunction and preferential multiplication of $B$-containing cells in the germ line tissues, as commonly found in the follicle cells of the testis in male grasshoppers, e.g., Locusta migratoria (Kayano, 1971).

(b) Meiotic drive whereby univalent $B$ s segregate at either the first or second division to be preferentially included in the functional meiotic products is known in plants and animals. In females of Myrmeleotettix, for example, the $B$ s are distributed on an asymmetrical spindle at first metaphase with a bias towards the egg side, and preferentially included in the secondary oocyte, rather than the polar body, at anaphase I (Hewitt, 1976). In Lilium callosum the majority of univalent $B$ s lie outside the metaphase I plate and in the direction of the micropylar end of the embryo sac, which includes the egg cell (Kayano, 1957). In the mealy bug, which has a "lecanoid" chromosome system and an inverted meiotic sequence, the $B$ s segregate preferentially into functional sperm nuclei at anaphase II ( Nur, 1962).

(c) Post-meiotic drive, as in rye, is the commonest system in plants and generally involves directed nondisjunction at the first pollen grain mitosis with inclusion of $B \mathrm{~s}$ in the generative nucleus. The system operating in rye with accumulation through both the male and female side is unique, as is that in maize where nondisjunction occurs at the second pollen grain division and would not in itself lead to accumulation without the element of selective fertilisation which favours male gametes containing the $B$ chromosomes (Roman, 1948). Further details of these accumulation mechanisms, and of some other lesser known ones, are described in Jones and Rees (1982).

In the wider context an analogy can be drawn between the drive mechanisms operating in $B$ chromosome systems and those found for some other kinds of genetic elements located within the $A$ chromosome complement. Mechanisms of meiotic drive in this wider sense, as reviewed by Zimmering et al. (1970), involve various components of the $A$ chromosome genome ranging from whole chromosomes, e.g., the $X$ chromosome in some Drosophila groups (Novitski et al., 1965); or parts of chromosomes, as in the preferential segregation to the functional megaspore of the large heterochromatic knob on abnormal chromosome 10 of maize (Rhoades, 1952), down to the level of the classic $t$ allele polymorphism in natural 
populations of the house mouse, Mus musculus (Dun, 1953; Lewontin, 1968). In homozygous recessive combinations the $t$ alleles are either lethal or they cause complete sterility-yet they are found in natural populations at high frequency. There is a meiotic drive mechanism which generate sperms containing $t$ alleles in excessive frequencies (85-99 per cent, Lewontin, 1968) from heterozygous males. The polymorphism is maintained by a balance between elimination of the alleles in inviable homozygotes and their accumulation through abnormal segregation at meiosis. The dynamics of the system have been presented by Lewontin (1968) and a more general population model of meiotic drive can be found in Hartl (1970). The $B$ chromosome system, while analogous to these other mechanisms of drive, differs in that the polymorphism involves dispensible supernumerary chromosomes which have no known function within the organism, no qualitative effects upon the phenotype, and which may be present in multiple copies in excess of the normal disomic number.

In terms of their redundancy, and their lack of phenotypic expression, the $B$ s in fact are much closer in their functional aspects and behaviour to what Orgel and Crick (1980) have defined as the "selfish DNA" component of the ( $A$ chromosome) genome, i.e., transposable elements, insertion sequences, middle repetitive sequences and other forms of so called junk DNAs. Orgel and Crick have defined selfish DNA as that fraction which can spread by forming additional copies of itself and which makes no specific contribution to the genome: examples are the $\mathrm{Ty}-1$ transposable element of yeast, which has no obvious phenotypic effect, which is present in some 35 dispersed copies and which comprises 2 per cent of the genome, and the middle repetitive sequences that make up 30 per cent of the eukaryote genome. Mechanisms of spread of these selfish DNAs have been discussed by Doolittle and Sapienza (1980) and their population dynamics is described by Ohta (1981).

This general debate about selfish DNA is a relatively recent affair (the last 10-15 years) and has its roots, according to Orgel and Crick, in Dawkins book on the "Selfish Gene" (Dawkins, 1976). We would respectfully like to suggest that the roots of this idea can be traced much further back than that, and can be found in the paper by Östergren, published in 1945, entitled ... "The parasitic nature of extra fragment chromosomes" ...i.e., $B$ chromosomes.

\section{REFERENCES}

BOSEMARK, N. O. 1956. On accessory chromosomes in Festuca pratensis. III. Frequency and geographical distribution of plants with accessory chromosomes. Hereditas, 42 , 189-210.

BOSEMARK, N. O. 1967. Edaphic factors and the geographical distribution of accessory chromosomes in Phleum phleoides. Hereditas, 57, 239-262.

BOUGOURD, S. M.. AND PARKER, J. S. 1979. The $B$ chromosome system of Allium schoenoprasum. II. Stability, inheritance and phenotypic effects. Chromosoma, 75, 369-383.

DAWKINS, R. 1976. The Selfish Gene. Oxford University Press.

DOOLITTLE, W. F., AND SAPIENZA, C. 1980. Selfish genes, the phenotype paradigm and genome evolution. Nature, 284, 601-603.

DUN, L. C. 1953. Variations in the segregation ratio as causes of variations of gene frequency. Acta Genet. Statist. Med., 4, 139-147.

FRÖsT, S. 1958. The geographical distribution of accessory chromosomes in Centaurea scabiosa. Hereditas, 44, 75-111. 
GRUN, P. 1959. Variability of accessory chromosomes in native populations of Allium cernuum. American J. Botany, 46, 218-224.

HAKKANSSON, A. 1948. Behaviour of accessory rye chromosomes in the embryo sac. Hereditas, 34, 35-59.

HARTL, D. L. 1970. Analysis of a general population genetic model of meiotic drive. Evolution, 24, 538-545.

HEWITT, G. M. $1973 a$. Variable transmission rates of a $B$ chromosome in Myrmeleotettix maculatus (Thunb.) Acrididae: Orthoptera. Chromosoma, 40, 83-106.

HEWITT, G. M. $1973 \mathrm{~b}$. The integration of supernumerary chromosomes into the Orthopteran genome. Cold Spring Harbor Symp. Quant, Biol., 38, 183-194.

HEWITT, G. M. 1976. Meiotic drive for $B$ chromosomes in the primary oocytes of Myrmeleotettix maculatus (Orthoptera: Acrididae). Chromosoma, 56, 381-391.

HEWITT, G. M., AND JOHN, B. 1967. The $B$ chromosome system of Myrmeleotettix maculatus (Thunb.). III. The statistics. Chromosoma, 21, 140-162.

HEWITT, G. M., AND JOHN, B. 1970. The $B$ chromosome system of Myrmeleotettix maculatus (Thunb.). IV. The dynamics. Evolution, 24, 169-180.

HEWITT, G., AND RUSCOE, C. 1971. Changes in microclimate correlated with a cline for $B$ chromosomes in the grasshopper Myrmeleotettix maculatus (Thunb.). (Orthoptera: Acrididae). J. Animal Ecology, 40, 753-765.

HUTCHINSON, J. 1975. Selection of $B$ chromosomes in Secale cereale and Lolium perenne. Heredity, 34, 39-52.

JONES, R. N. 1975. $B$ chromosome systems in flowering piants and animal species. International Review of Cytology, 40,1-100.

JONES, R. N., AND REES, H. 1967. Genotypic control of chromosome behaviour in rye. XI. The influence of $B$ chromosomes on meiosis. Heredity, 22, 333-347.

JONES, R. N., AND REES, H. 1968. The influence of $B$ chromosomes upon the nuclear phenotype in rye. Chromosoma, 24, 158-176.

JONES, R. N., AND REES, H. 1969. An anomalous variation due to $B$ chromosomes in rye. Heredity, 24, 265-271.

JONES, R. N., AND REES, H. 1982. B chromosomes. Academic Press.

KAYANO, H. 1957. Cytogenetic studies in Lilium callosum. III. Preferential segregation of a supernumerary chromosome in EMC's. Proc. Japan Acad., 33, 553-558.

KAYANO, H. 1971. Accumulation of $B$ chromosomes in the germ line of Locusta migratoria. Heredity, 27, 119-123.

KIMURA, M., AND KAYANO, H. 1961. The maintenance of supernumerary chromosomes in wild populations of Lilium callosum by preferential segregation. Genetics, 46, 16991712.

KISHIKAWA, H. 1965. Cytogenetic studies of $B$ chromosomes in rye, Secale cereale L., in Japan. Agricultural Bulletin of Saga University, 21, 1-81.

KISHIKAWA, H. 1970. Effects of temperature and soil moisture on frequency of accessory chromosomes in rye, Secale cereale L. Japanese J. Breeding, 20, 269-274.

KRANZ, A. R. 1963. Beiträge zur cytologischen und genetischen evolutionsforschung an dem roggen. Z. Pflanzenzüchtung, 50, 44-58.

LEE, W. J., AND MIN, B. R. 1965a. Frequency and geographical distribution of rye with accessory chromosomes in Korea. Wheat Information Service, 21, $27-28$.

LEE, W. J., AND MIN, B. R. $1965 b$. On accessory chromosomes in Secale cereale. I. Frequency and geographical distribution of plants with accessory chromosomes in Korea. Korean J. Botany, 8, 1-6.

LEWONTIN, R. C. 1968. The effect of differential viability on the population dynamics of $t$ alleles in the house mouse. Evolution, 22, 262-273.

LIMA-DE-FARIA, A. 1963. The evolution of the structural pattern in a rye $B$ chromosome. Evolution, 17, 289-295.

MATThews, R. B. 1981. Studies on B Chromosomes with Particular Reference to Plant Disease. Ph.D. Thesis, University of Wales.

MÜNTZING, A. 1943. Genetical effects of duplicated fragment chromosomes in rye. Hereditas, 29, 91-112.

MÜNTZING, A. 1945. Cytological studies of extra fragment chromosomes in rye. II. Transmission and multiplication of standard fragments and iso-fragments. Hereditas, 31 , 457-477.

MÜNTZING, A. 1946. Cytological studies of extra fragment chromosomes in rye. III. The mechanism of nondisjunction at the pollen mitosis. Hereditas, 32, 97-119. 
MÜNTZING, A. 1950. Accessory chromosomes in rye populations from Turkey and Afghanistan. Hereditas, 36, 507-509.

MÜNTZING, A. 1957 a. Frequency of accessory chromosomes in rye strains from Korea. Wheat Information Service, 5, 16-18.

MÜNTZING, A. $1957 b$. Frequency of accessory chromosomes in rye strains from Iran and Korea. Hereditas, 43, 682-685.

NOVITSKI, E., PEACOCK, W. J., AND ENGEL, J. 1965. Cytological basis of sex ratio in Drosophila pseudoobscura. Science, 148, 516-517.

NUR, U. 1962. A supernumerary chromosome with an accumulation mechanism in the lecanoid genetic system. Chromosoma, 13, 249-271.

NUR, U. 1966. Harmful supernumerary chromosomes in a mealy bug population. Genetics, $54,1225-1238$.

NUR, U. 1969. Harmful $B$ chromosomes in a mealy bug: additional evidence. Chromosoma, $28,280-297$.

NUR, U. 1977. Maintenance of a "parasitic" $B$ chromosome in the grasshopper Melanoplus femur-rubrum. Genetics, 87, 499-512.

OHTA, T. 1981. Population genetics of selfish DNA. Nature, 292, 648-649.

ORGEL, L. E., AND CRICK, F. H. C. 1980. Selfish DNA: the ultimate parasite. Nature, 284, 604-607.

ÖSTERGREN, B. 1945. Parasitic nature of extra fragment chromosomes. Botaniska Notiser, 2, 157-163.

PUERTAS, M. J., AND CARMONA, R. 1976. Greater ability of pollen tube growth in rye plants with $2 B$ chromosomes. Theoret. Appl. Genet., 47, 41-43.

PUERTAS, M. J., AND LACADENA, J. R. 1974. The transmission of rye $B$ chromosomes in natural pollination. Theoret. Appl. Genet., 45, 197-204.

REES, H., AND HUTCHINSON, J. 1973. Nuclear DNA variation due to $B$ chromosomes. Cold Spring Harbor Symposium on Quantitative Biology, 38, 175-182.

RHOADES, M. M. 1952. Preferential segregation in maize. In Heterosis, Iowa State College Press, Chap. 4.

ROMAN, H. 1948. Directed fertilisation in maize. Proc. Natl. Acad. Sci., 34, 36-42.

ZECĚVIĆ, L., AND PAUNOVIĆ, D. 1967. B chromosome frequency in Yugoslav rye populations. Biologia Plantarum 9, 205-211.

ZIMMERING, S., SANDLER, L., AND NICOLETTI, B. 1970. Mechanisms of meiotic drive. Ann. Rev. Genet., 4, 409-436. 